\title{
Nanosecond Optical Parametric Oscillator Based On Highly-Nonlinear Dispersion-Shifted Fiber
}

\author{
Kim K. Y. Cheung, Yue Zhou, and Kenneth K. Y. Wong \\ Photonic Systems Research Laboratory, Department of Electrical and Electronic Engineering, \\ The University of Hong Kong, Pokfulam Road, Hong Kong \\ Phone: +(852) 2857 8483, Fax: +(852) 2559 8738, Email: kywong@eee.hku.hk
}

\begin{abstract}
We demonstrate a fully fiber-integrated tunable nanosecond optical parametric oscillator based on 50-m highly-nonlinear fiber with tuning range from $1433 \mathrm{~nm}$ to $1490 \mathrm{~nm}$ and from $1625 \mathrm{~nm}$ to $1685 \mathrm{~nm}$ by tuning the pump wavelength between $1549 \mathrm{~nm}$ and $1555 \mathrm{~nm}$.
\end{abstract}

\section{Introduction}

Fiber-based optical parametric oscillators (FOPO) which operated through four-wave missing (FWM) mediated on $\chi^{(3)}$ nonlinearity of glass have received considerable attention recently due to the ultra-fast response, widegain bandwidth [1], high gain [2] and large detune from the pump [1] of fiber optical parametric amplifier (FOPA). Due to the requirement of watt-level pump powers, most of FOPOs were operated with picosecond pulsed $[3,4]$ or even femtosecond pulsed $[5,6]$ pumps. As the round trip time of signal should match to the pump repetition rate, cavity length should be adjusted to synchronize the signal with pump. Pulses with different wavelengths experience different dispersion so as the round trip time of signal also varied. As the pulse-width of both femtosecond and picosecond pulses are narrow, changing of pump wavelength will cause misalignment between pump and signal, strict synchronization between signal and pump pulses is needed and cavity length is required to adjust every time when tuning the pump wavelength. For continuous-wave (CW) FOPO [7, 8], adjustment on cavity is not required but the pump power required will be higher. Moreover for $\mathrm{CW}$ FOPO, phase modulation (PM) on pump is always required to suppress the stimulated Brillouin scattering (SBS) effect, the pump PM can cause a large power fluctuation due to the PM-to-IM conversion in the amplified and wavelengthconverted signals [9]. For nanosecond pulse [10], suppression of SBS is not required and also due to a relatively large pulse width, pump and signal will overlap with each other even when the pump wavelength changes. Therefore, changing cavity length is not required and this will give flexibility in tuning the pump wavelength. In this paper we propose and demonstrate a nanosecond fiber OPO based on highly-nonlinear dispersion-shifted fiber (HNL-DSF) with a setup similar to a previously reported picosecond pulsed pump OPO [11]. Pump wavelength can be varied flexibly without changing the cavity length. The tuning range is from $1433 \mathrm{~nm}$ to $1490 \mathrm{~nm}$ and from $1625 \mathrm{~nm}$ to $1685 \mathrm{~nm}$ by tuning the pump wavelength from $1549 \mathrm{~nm}$ and $1555 \mathrm{~nm}$

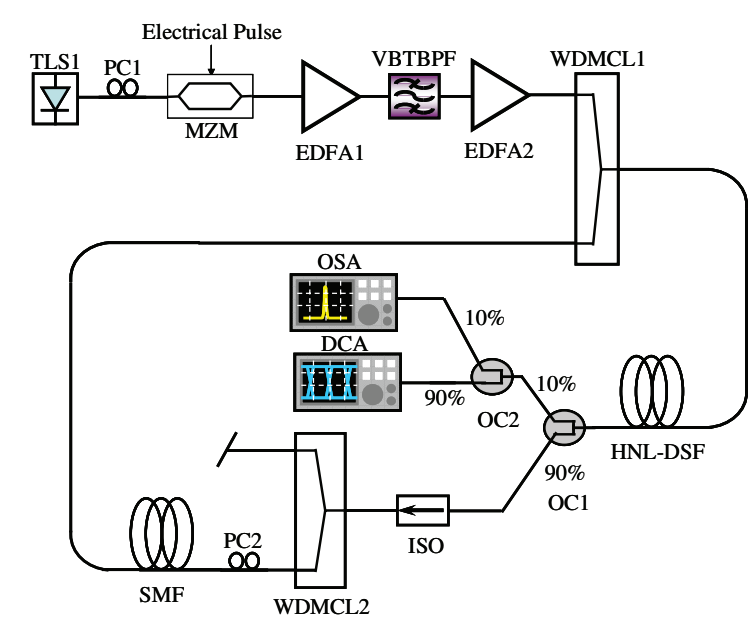

Fig. 1. Experimental setup of FOPO

and the average power required is $50 \mathrm{~mW}$.

\section{Experimental Setup}

The experimental setup of the FOPO is shown in Fig. 1. The pump is generated by a CW tunable laser source (TLS) with wavelength tuning from $1549 \mathrm{~nm}$ to 1555 $\mathrm{nm}$. It is then modulated with 25 -ns pulses at a repetition frequency of $600 \mathrm{kHz}$ by a Mach-Zehnder intensity modulator (MZM). The polarization controller (PC), $\mathrm{PC} 1$, is used to align the state of polarization (SOP) of the pump to the MZM. The modulated pump is then amplified by two-stage erbium-doped fiber amplifiers (EDFAs), EDFA1 and EDFA2, with variable bandwidth tunable bandpass filter (VBTBPF) inserted between the two EDFAs to remove amplified spontaneous emission (ASE) noise. The bandwidth of VBTBPF is $8 \mathrm{~nm}$ so that tuning filter is not required even the pump wavelength is changed. The average power of the pump measures after the EDFA2 is $50 \mathrm{~mW}$ and the corresponding pump peak power is about $3.3 \mathrm{~W}$. The pump is then coupled into the cavity for parametric amplification through the wavelength-division multiplexing coupler (WDMCL1) which has a cutoff wavelength at $1565 \mathrm{~nm}$. The 50-m HNL-DSF used has here a nonlinear coefficient of $14 \mathrm{~W}^{-}$ ${ }^{1} \mathrm{~km}^{-1}$, zero-dispersion wavelength (ZDW) at $1554.7 \mathrm{~nm}$ and dispersion slope of $0.035 \mathrm{ps} / \mathrm{nm}^{2} / \mathrm{km}$. A $10 / 90$ optical coupler (OC1) in the cavity provided $90 \%$ feedback and $10 \%$ output. An isolator (ISO) inserted inside the cavity ensures uni-directional operation and prevents oscillating by back reflection. WDMCL2 with 
cutoff wavelength at $1565 \mathrm{~nm}$ is used to remove the pump and anti-Stokes wave so that only the signal (Stokes wave) returns to the HNL-DSF through WDMCL1. As a result, the FOPO is only singly resonant with the signal. The PC2 in the cavity is used to align the signal's SOP with the pump so as to maximize the signal gain. In order to make the round trip time of the signal matches the pump repetition rate, about 250-m singlemode fiber (SMF) is added to synchronize the signal with the pump. The FOPO output lights are monitored by an optical spectrum analyzer (OSA) and a digital communication analyzer (DCA) with $10-\mathrm{GHz}$ bandwidth through OC2.

\section{Results and Discussion}

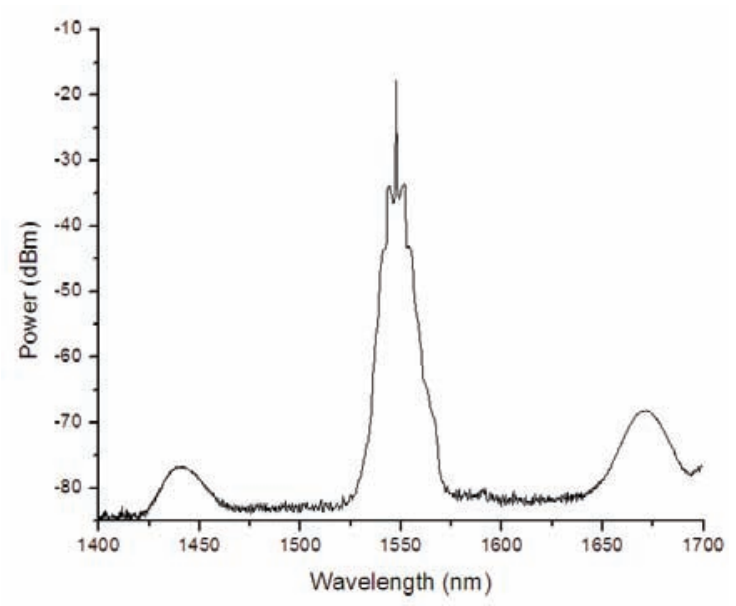

Fig. 2. Spontaneous emission spectrum of OPA.

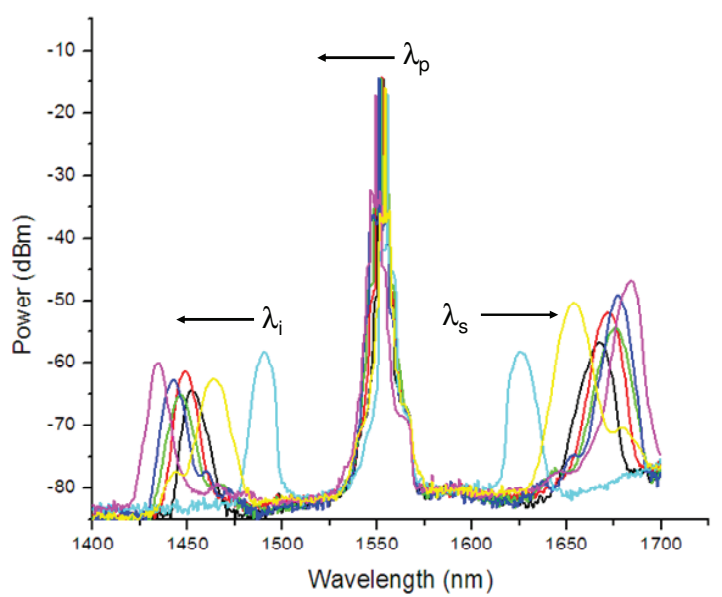

Fig. 3. Signal output spectrum of FOPO. The pump is tuned between $1549 \mathrm{~nm}$ and $1555 \mathrm{~nm}$.

Fig. 2 shows the OPA spectrum measures after the HNLDSF with pump wavelength at $1550 \mathrm{~nm}$. It can be observed that there are two discrete narrow sidebands located $1433 \mathrm{~nm}$ to $1490 \mathrm{~nm}$ (A band) and $1625 \mathrm{~nm}$ to $1685 \mathrm{~nm}$ (B band). Fig. 3 shows the spontaneous emission spectra from OPA where the pump pulse width is $25-\mathrm{ns}$ and pump wavelength is tuned from $1555 \mathrm{~nm}$ to $1549 \mathrm{~nm}$. The pump power is slightly adjusted to maintain narrow linewidth for all spectra. The pedestal of the pump spectrum is due to the wide bandwidth of VBTBPF located between the two EDFAs. When the pump wavelength is tuned away from ZDW to the shorter wavelength, the output oscillating wavelength shifted gradually away from the pump. The maximum separation of $107 \mathrm{~nm}$ between pump and oscillating sideband is attained when the pump was at $1550 \mathrm{~nm}$. The achievable output tuning range is from $1433 \mathrm{~nm}$ to 1490 $\mathrm{nm}$ and from $1625 \mathrm{~nm}$ to $1685 \mathrm{~nm}$, which is over $110 \mathrm{~nm}$ with $6 \mathrm{~nm}$ of pump tuning. Linewidth of oscillating wavelengths is about $8 \mathrm{~nm}$ and tuning range may be limited by fluctuation of ZDW. The asymmetry of the spectra is due to the Raman effect occurred on the B band which cause the signals on the $\mathrm{B}$ band side experience higher gain than that on $\mathrm{A}$ band. As the repetition rate of pump pulse used is $600 \mathrm{kHz}$, about 250-m SMF is inserted to synchronize the pump and signal. Since the cavity length, including HNLF-DSF, is longer than $300 \mathrm{~m}$, multiple modes oscillate inside the cavity simultaneously.

\section{Conclusion}

In conclusion, an all-fiber nanosecond optical parametric oscillator based on HNL-DSF is demonstrated. The output tuning range is over $110 \mathrm{~nm}$ with $6 \mathrm{~nm}$ pump tuning. By tuning the pump wavelengths, the sidebands on A and B band will move away. Since changing the cavity length and tuning of VBTBPF is not required in this scheme, it could be found potentially useful in generating the sweeping source by simply tuning the pump wavelength.

\section{Acknowledgment}

The work described in this paper was partially supported by grants from the Research Grants Council of the Hong Kong Special Administrative Region, China (Project No. HKU 7172/07E and HKU 7179/08E). The authors would also like to acknowledge Sumitomo Electric Industries for providing the HNL-DSF and Alnair Laboratories for providing the VBTBPF.

\section{References}

1. M. E. Marhic et al, IEEE J. Sel. Top. Quantum Electron. 10 (2004) p.1133

2. T. Torounidis et al, J. Lightwave Technol. 23 (2005) p.4067

3. J. Lasri et al, IEEE Photon. Technol. Lett. 15 (2003) p. 1058

4. D. K. Serkland et al, Opt. Lett. 24 (1999) p. 92

5. J. E. Sharping el al, Opt. Express. 16 (2008) p. 18050

6. Y. Deng et al, Opt. Lett. 30 (2005) p. 1234

7. M. E. Marhic et al, Opt. Lett. 27 (2002) p. 1439

8. C. de Matos et al, Opt. Lett. 29 (2004) p. 983

9. R. Jiang et al, OFC. Paper OWB2 (2007)

10. G. K. L. Wong et al, Opt. Express. 16 (2007) p. 2947

11. Y. Zhou et al, Opt. Lett. 34 (2009) p. 989 